\title{
Theta correspondence and Hecke operators relative to a quadratic extension
}

\author{
by
}

\author{
ZE-Li Dou (Fort Worth, TX)
}

To Professor Goro Shimura, with admiration and gratitude

Introduction. Let $E / F$ be a totally real quadratic extension of a totally real algebraic number field. To a suitably defined automorphic form $h$ defined with respect to a quaternion algebra $B_{E}$ over $E$, we can associate a Hilbert modular form $I(z, h)$ defined with respect to the field $F$. Such a lifting has been explicitly considered in the author's recent paper [D99], via a convolution with a theta function, and the Fourier coefficients of the theta lift have been computed in terms of certain periods of the original form. The purpose of the current paper is to establish an explicit formula relating the actions of the Hecke operators on the original automorphic form and its theta lift. This result is analogous to theorems of Shimura in, for example, [Sh82] and [Sh88], where he has shown commutativity properties with respect to these Hecke operators. In addition to their inherent interest, these commutativity results are necessary in obtaining algebraicity results concerning Shimura's period invariants as well as certain special $L$-values which naturally arise. The reader can consult Shimura's [Sh88] for further motivation in this regard. See also [Y]. It is the author's hope to discuss applications in this direction in the near future.

Since it is essential for our purposes to consider everything explicitly, the quantity of technical details is considerable. In the interest of space, and also in order not to obscure essential ideas, a large amount of computation has been suppressed. Instead we have endeavored to point out key ingredients in the computations and to give precise references. It is then hoped that

2000 Mathematics Subject Classification: 11F27, 11F32, 11F41, 11F55, 11F60.

Key words and phrases: Hilbert modular forms, quaternionic automorphic forms, theta functions, theta correspondence, Hecke operators.

Research for this article was partially supported by NSA Grant MDA904-97-1-0109 and by the Texas Christian University Research Fund. 
the abbreviated calculations can be filled out, when necessary, without too much difficulty. Also, relevant facts from [D99] are briefly recalled in this paper as the need occurs, so that this paper can be read independently of [D99].

This paper is dedicated to the author's former thesis advisor, Professor Goro Shimura. Professor Shimura has on many occasions shared his insights with the author in the most generous manner. Indeed, he proposed the current project to the author in the first place, and even allowed him access to some of his unpublished notes ([Sh]). Most fundamentally, the author is grateful to him for his continuous interest in the author's general well-being. The author also thanks Professors A. W. Bluher, K. Kramer, B. Palka, and H. Yoshida for their advice and help. Further, he is grateful for the corrections and suggestions he received from the referee. Finally, the author thanks his family for their steadfast support and unwavering confidence.

1. Automorphic forms on $H^{\zeta}$ and on $\mathcal{G}_{\mathbb{A}}^{\prime}$. We begin by establishing notation and recalling the definition of the automorphic forms for which a theta lift has been constructed in [D99]. Since the details can be found there, we give here only a very brief account; in the meantime we establish some notation.

Let $F$ be a totally real algebraic number field, and let $E / F$ be a totally real quadratic extension. Let $B_{E}$ be a quaternion algebra over $E$ equipped with an $F$-linear automorphism $\tau$ such that $\tau^{2}=\operatorname{id}_{B_{E}}$, but $\left.\tau\right|_{E} \neq \operatorname{id}_{E}$. The main involution of $B_{E}$ will be denoted by ${ }^{*}$. We consider the following subsets of $B_{E}$ :

$$
B=\left\{x \in B_{E} \mid x^{\tau}=x\right\} \quad \text { and } \quad V=\left\{x \in B_{E} \mid x^{\tau}=-x^{*}\right\} .
$$

Then $B$ is a quaternion algebra over $F$, and $V$ is a vector space over $F$ of dimension 4. As usual, we define the norm and trace by the formulas $\mathrm{N}(x)=x x^{*}$ and $\operatorname{Tr}(x)=x+x^{*}$. It is easy to check that, on the vector space $V, \mathrm{~N}$ is an $F$-valued quadratic form. Moreover, given any $a \in B_{E}, V$ is stable under the mapping $x \mapsto a^{\tau} x a^{*}$, and we have the formula

$$
\mathrm{N}\left(a^{\tau} x a^{*}\right)=\mathrm{N}^{\prime}(a) \mathrm{N}(x), \quad \text { where } \quad \mathrm{N}^{\prime}(a):=\mathrm{N}_{E / F}(\mathrm{~N}(a)) .
$$

Our theory is essentially independent of the choice of the automorphism $\tau$, as explained in Proposition 1.1 of [D99].

Denote the set of archimedean primes in $F$ by $\mathbf{a}$, and the set of finite primes by $\mathbf{h}$. The set of archimedean primes in $E$ is written $J(E)$. Let $\delta$ denote the set of primes $v \in \mathbf{a}$ which are unramified in $B$, and $\delta^{\prime}=$ $\mathbf{a}-\delta$ the set of those ramified in $B$. The subsets of $J(E)$ consisting of extensions of primes in $\delta$ and $\delta^{\prime}$ are denoted by $\zeta$ and $\zeta^{\prime}$, respectively. Using these notations, we have $\mathbb{R}$-linear isomorphisms $B_{\mathbf{a}} \cong \mathrm{M}_{2}(\mathbb{R})^{\delta} \times \mathbb{H}^{\delta^{\prime}}$ and 
$\left(B_{E}\right)_{\mathbf{a}} \cong \mathrm{M}_{2}(\mathbb{R})^{\zeta} \times \mathbb{H}^{\zeta^{\prime}}$. Here $\mathbb{H}$ denotes the ring of Hamilton quaternions, and the subscript $\mathbf{a}$ indicates the infinite part of the adelized space under consideration. The adelization itself will be denoted by the subscript $\mathbb{A}$, and its finite part will be given the subscript $\mathbf{h}$. For each $v \in \mathbf{a}$, we fix, once and for all, an extension $u \in J(E)$. The collection of these chosen $u$ is written $\iota$. Further, we denote by $\eta$ and $\eta^{\prime}$, respectively, the subsets of $\iota$ corresponding to $\delta$ and $\delta^{\prime}$. Throughout this paper, we shall assume $\zeta \neq \emptyset$.

For every $m \geq 0$, there is an $\mathbb{R}$-rational irreducible polynomial representation $\sigma_{m}: \mathbb{H}^{\times} \rightarrow \mathrm{GL}_{m+1}(\mathbb{C})$ of degree $m$, which is unique up to equivalence. By fixing suitable isomorphisms for $B_{\mathbf{a}}$ and $\left(B_{E}\right)_{\mathbf{a}}$, we may assume that $\sigma_{m}$ respects the $\overline{\mathbb{Q}}$-structure. If $k \in \mathbb{Z}^{\iota}$ is a weight such that $k_{u} \geq 1$ for $u \in \eta$ and $k_{u} \geq 2$ for $u \in \eta^{\prime}$, then we define a representation on $\left(B_{E}^{\times}\right)_{\mathbb{A}}$ by

$$
\sigma(\alpha)=\bigotimes_{u \in \eta^{\prime}} \sigma_{k_{u}-2}\left(\alpha_{u}\right), \quad \forall \alpha \in\left(B_{E}^{\times}\right)_{\mathbb{A}} .
$$

The representation space for $\sigma$ will be denoted by $\mathcal{X}$. It may be identified with $\bigotimes_{u \in \eta^{\prime}} \mathbb{C}^{k_{u}-1}$.

To define the factor of automorphy in the definition of automorphic forms, we need some more notation. Let

$$
\begin{aligned}
\mathcal{G}^{\prime} & =\left(B_{E}\right)^{\times}, & \mathcal{G}_{\mathbf{a}+}^{\prime} & =\left\{x \in \mathcal{G}_{\mathbf{a}}^{\prime} \mid \mathrm{N}(x) \gg 0\right\}, \\
\mathcal{G}_{\mathbb{A}+}^{\prime} & =\left\{x \in \mathcal{G}_{\mathbb{A}}^{\prime} \mid x_{\mathbf{a}} \in \mathcal{G}_{\mathbf{a}+}^{\prime}\right\}, & \mathcal{G}_{1}^{\prime} & =\left\{x \in \mathcal{G}^{\prime} \mid \mathrm{N}(x)=1\right\} .
\end{aligned}
$$

Thus the representation in (1) is $\sigma: \mathcal{G}_{\mathbb{A}}^{\prime} \rightarrow \operatorname{GL}(\mathcal{X})$. For $\alpha \in \mathcal{G}_{\mathbb{A}}^{\prime}$ and $w \in H^{\zeta}$, we put

$$
\alpha w=\alpha(w)=\left(\alpha_{u} w_{u}\right)_{u \in \zeta}=\left(\frac{a_{u} w_{u}+b_{u}}{c_{u} w_{u}+d_{u}}\right)_{u \in \zeta}
$$

and

$$
j(\alpha, w)=\left(j\left(\alpha_{u}, w_{u}\right)\right)_{u \in \zeta}=\left(\left|\operatorname{det}\left(\alpha_{u}\right)\right|^{-1 / 2}\left(c_{u} w_{u}+d_{u}\right)\right)_{u \in \zeta},
$$

where $a_{u}, b_{u}, c_{u}$ and $d_{u}$ are the entries of $\alpha_{u}$ in the standard order.

We now define $\operatorname{End}(\mathcal{X})$-valued holomorphic automorphic forms of weight $k+\tau k$ on $H^{\zeta}$, where $k \in \mathbb{Z}^{\iota}$ as above. Given a mapping $f: H^{\zeta} \rightarrow \operatorname{End}(\mathcal{X})$, we define another mapping of the same kind, denoted by $f \|_{k+\tau k} \alpha$ and sometimes simply $f \| \alpha$, by the following formula:

$$
\left(f \|_{k+\tau k} \alpha\right)(w)=j(\alpha, w)^{-k \eta-\tau(k \eta)} \sigma\left(\mathrm{N}(\alpha)^{1 / 2} \alpha^{-1}\right) f(\alpha w) \sigma\left(\mathrm{N}(\alpha)^{-\tau / 2} \alpha^{\tau}\right) .
$$

Let $\Gamma$ be a congruence subgroup of $\mathcal{G}_{1}^{\prime}$. The space of holomorphic automorphic forms of weight $k+\tau k$ with respect to $\Gamma$ is the set of all holomorphic mappings $f: H^{\zeta} \rightarrow \operatorname{End}(\mathcal{X})$ such that $f \| \alpha=f$ for all $\alpha \in \Gamma$, and also the usual cusp condition in the case where $B_{E}=\mathrm{M}_{2}(E)$. This space is denoted by $\mathcal{S}_{k+\tau k}(\Gamma)$. The union of such spaces over all congruence subgroups is denoted by $\mathcal{S}_{k+\tau k}\left(B_{E}\right)$. Denoting by $d_{H}^{\zeta} w$ the Haar measure on $H^{\zeta}$, we define 
an inner product of two $\mathbb{C}^{\infty}$-mappings $f$ and $g$ of $H^{\zeta}$ into $\operatorname{End}(\mathcal{X})$, such that $f \| \alpha=f$ and $g \| \alpha=g$ for all $\alpha \in \Gamma$ for some congruence subgroup $\Gamma$, by the formula

$$
\langle f, g\rangle=\operatorname{vol}(D)^{-1} \int_{D} \operatorname{Tr}\left(\overline{{ }^{t} f(w)} g(w)\right) \operatorname{Im}(w)^{k \eta+\tau(k \eta)} d_{H}^{\zeta} w .
$$

Here $D:=\Gamma \backslash H^{\zeta}$, and $\operatorname{vol}(D):=\int_{D} d_{H}^{\zeta} w$. This definition is independent of the choice of $\Gamma$.

We now consider adelic automorphic forms defined on $\mathcal{G}_{\mathbb{A}}^{\prime}$. Let $\mathfrak{g}$ and $\mathfrak{g}^{E}$ denote the rings of integers in $F$ and in $E$, respectively. Denote by $\mathfrak{d}_{B}$ the product of all finite primes of $F$ which are ramified in $B$, and by $\mathfrak{d}_{B}^{E}$ the product of all finite primes of $E$ which are ramified in $B_{E}$. It is not difficult to check that, if $v$ is a finite place of $F$ which is inert in $E$, then the place in $E$ lying over $v$ is unramified in $B_{E}$. Thus a prime $u$ of $E$ divides $\mathfrak{d}_{B}^{E}$ if and only if it divides $\mathfrak{d}_{B}$ and splits over $F$. We note that there exists a maximal order $\mathfrak{o}$ in $B_{E}$ which contains a maximal order of $B$, such that we can find, for every $v \in \mathbf{h}$ prime to $\mathfrak{d}_{B}^{E}$, an $E_{v}$-linear isomorphism $\mu_{v}:\left(B_{E}\right)_{v} \rightarrow \mathrm{M}_{2}\left(E_{v}\right)$ with the property $\mu_{v}\left(\mathfrak{o}_{v}\right)=\mathrm{M}_{2}\left(\mathfrak{g}_{v}^{E}\right)$. Moreover, if $v \nmid \mathfrak{d}_{B}$, then $\mu_{v}\left(x^{\tau}\right)=\mu_{v}(x)^{\tau}$, where we understand that $\tau$ acts entry-wise on $\mathrm{M}_{2}\left(E_{v}\right)$. A proof of this fact can be found in [D98]. We shall from now on fix $\mathfrak{o}$ and $\mu_{v}$ as above.

Let $\mathfrak{m}$ be an integral ideal in $E$. We define an order of level $\mathfrak{m}$ to be the $\mathfrak{g}^{E}$-lattice $\mathfrak{o}_{1} \subset B_{E}$ given by

(i) $\mathfrak{o}_{1 v}=\mathfrak{g}_{v}^{E}+\mathfrak{m o}_{v}$ if $v \mid \mathfrak{d}_{B}^{E}$;

(ii) $\mathfrak{o}_{1 v}=\mu_{v}^{-1}\left(\left\{x \in \mathrm{M}_{2}\left(E_{v}\right) \mid a_{x} \in \mathfrak{g}_{v}^{E}, b_{x} \in \mathfrak{d}^{-1} \mathfrak{g}_{v}^{E}, c_{x} \in \mathfrak{d} \mathfrak{m}_{v}, d_{x} \in \mathfrak{g}_{v}^{E}\right\}\right)$ if $v \nmid \mathfrak{d}_{B}^{E}$.

Here $\mathfrak{d}$ denotes the different of $F$ over $\mathbb{Q}$. Given such an $\mathfrak{m}$, put

$$
W_{\mathfrak{m}}^{\prime}=\mathcal{G}_{\mathbf{a}+}^{\prime} \prod_{v \in \mathbf{h}} \mathfrak{o}_{1 v}^{\times},
$$

where $\mathcal{G}_{\mathbf{a}+}^{\prime}:=\left\{x \in \mathcal{G}_{\mathbf{a}}^{\prime} \mid \mathrm{N}(x) \gg 0\right\}$. When $\mathfrak{m}$ is understood, we sometimes write $W^{\prime}$ to shorten notation. There exists a finite subset $Q^{\prime} \subset \mathcal{G}_{\mathbf{h}}^{\prime}$ such that we have a coset decomposition

$$
\mathcal{G}_{\mathbb{A}}^{\prime}=\bigsqcup_{q \in Q^{\prime}} \mathcal{G}^{\prime} q W_{\mathfrak{m}}^{\prime}
$$

Finally, let $\Phi$ be a Hecke character of $E$ such that the conductor of $\Phi$ is prime to $\mathfrak{d}_{B}^{E}$ and is a divisor of $\mathfrak{m}$, and, in addition,

$$
\Phi_{\mathbf{a}}(x)=\operatorname{sgn}\left(x_{\mathbf{a}}\right)^{k+\tau k}\left|x_{\mathbf{a}}\right|^{2 i \kappa},
$$

where $\kappa \in E_{\mathbf{a}}=\mathbb{R}^{J(E)}$, and $\|\kappa\|=0$. Here $\|\kappa\|$ denotes the sum of the components of $\kappa$. 
The space of adelic automorphic forms $\mathcal{S}_{k+\tau k}\left(\mathfrak{m}, \Phi ; B_{E}\right)$ is now defined to be the set of all mappings $\mathbf{g}: \mathcal{G}_{\mathbb{A}}^{\prime} \rightarrow \operatorname{End}(\mathcal{X})$ satisfying the following three conditions:

(a) $\mathbf{g}(s x)=\Phi(s) \mathbf{g}(x), \quad \forall s \in E_{\mathbb{A}}^{\times}, \forall x \in \mathcal{G}_{\AA}^{\prime}$.

(b) $\mathbf{g}(\beta x w)=\Phi_{\mathfrak{m}}\left(d_{w}\right) \mathbf{g}(x), \quad \forall \beta \in \mathcal{G}^{\prime}, \forall w \in W^{\prime}, w_{\mathbf{a}}=1, \forall x \in \mathcal{G}_{\mathbb{A}}^{\prime}$.

(c) For every $p \in \mathcal{G}_{\mathbf{h}}^{\prime}$, there is an element $g_{p}$ of $\mathcal{S}_{k+\tau k}\left(B_{E}\right)$ such that

$$
\mathbf{g}(p y)=\Phi(\mathrm{N}(p)) \mathrm{N}(y)^{i \kappa}\left(g_{p} \|_{k+\tau k} y\right)(\mathbf{i}), \quad \forall y \in \mathcal{G}_{\mathbf{a}+}^{\prime} .
$$

As usual, we have here $\mathbf{i}=(i, i, \ldots, i) \in H^{\zeta}, \Phi_{\mathfrak{m}}=\prod_{u \mid \mathfrak{m}} \Phi_{u}$, while $d_{w} \in E_{\mathbf{h}}^{\times}$ is defined as follows. The $v$-component of $d_{w}$ is 1 for all $v$ except when $v \mid \mathrm{N}(\mathfrak{m})$ and $v$ is prime to $\mathfrak{d}_{B}^{E}$, in which case it is defined to be the $d$-entry of $\mu_{v}(w)$. We sometimes also write $d(w)$, etc., as in (4) below.

Let $\Phi$ and $\mathfrak{m}$ be given as above, and choose a $\kappa$ such that (3) holds. For each $p \in \mathcal{G}_{\mathbf{h}}^{\prime}$, we put

$$
\Delta_{p}^{\prime}=p W^{\prime} p^{-1} \cap \mathcal{G}^{\prime} .
$$

We then define a subspace of $\mathcal{S}_{k+\tau k}\left(B_{E}\right)$, denoted by $C\left(\Delta_{p}^{\prime}, \Phi_{\mathfrak{m}}, \kappa\right)$, to be the set of all elements of $\mathcal{S}_{k+\tau k}\left(B_{E}\right)$ such that

$$
h \|_{k+\tau k} \gamma=\Phi_{\mathfrak{m}}\left(a\left(p^{-1} \gamma p\right)\right) \mathrm{N}(\gamma)^{i \kappa} h, \quad \forall \gamma \in \Delta_{p}^{\prime} .
$$

With respect to the coset decomposition (2) we have an embedding

$$
\mathcal{S}_{k+\tau k}\left(\mathfrak{m}, \Phi, B_{E}\right) \hookrightarrow \prod_{q \in Q^{\prime}} C\left(\Delta_{q}^{\prime}, \Phi_{\mathfrak{m}}, \kappa\right) .
$$

For a given form $\mathbf{g} \in \mathcal{S}_{k+\tau k}\left(\mathfrak{m}, \Phi, B_{E}\right)$, the embedding above is defined by condition (c) above, with the $p$ there replaced by the various $q \in Q^{\prime}$. It is straightforward to check that $g_{q} \in C\left(\Delta_{q}^{\prime}, \Phi_{\mathfrak{m}}, \kappa\right)$ for every $q \in Q^{\prime}$. We remark that our definition of $C\left(\Delta_{p}^{\prime}, \Phi_{\mathfrak{m}}, \kappa\right)$ is analogous to Shimura's $\mathcal{S}_{k}\left(\widetilde{\Gamma}_{p}, \phi, \lambda\right)$ in [Sh91]. Some further properties which parallel those of $\mathcal{S}_{k}\left(\widetilde{\Gamma}_{p}, \phi, \lambda\right)$ can be proved for $C\left(\Delta_{p}^{\prime}, \Phi_{\mathfrak{m}}, \kappa\right)$, but are omitted here.

Naturally, if $\mathbf{f}=\left(f_{q}\right)_{q \in Q^{\prime}}$ and $\mathbf{g}=\left(g_{q}\right)_{q \in Q^{\prime}}$ are in $\mathcal{S}_{k+\tau k}\left(\mathfrak{m}, \Phi, B_{E}\right)$, then their inner product is defined by

$$
\langle\mathbf{f}, \mathbf{g}\rangle=\left\|Q^{\prime}\right\|^{-1} \sum_{q \in Q^{\prime}}\left\langle f_{q}, g_{q}\right\rangle .
$$

Finally, it is also necessary to consider automorphic forms with respect to a subgroup $\mathcal{G}$ of $\mathcal{G}^{\prime}$ defined by

$$
\mathcal{G}=\left\{x \in \mathcal{G}^{\prime} \mid \mathrm{N}(x) \in F\right\} .
$$

The significance of this possibility is that the vector space $V$ is stable under the mapping $x \mapsto \alpha x \alpha^{-\tau}$ for every $\alpha \in \mathcal{G}$, as can be easily checked. Put

$$
W=W_{\mathfrak{m}}=W_{\mathfrak{m}}^{\prime} \cap \mathcal{G}_{\mathbb{A}} \quad \text { and } \quad \mathcal{G}_{+}=\mathcal{G} \cap \mathcal{G}_{+}^{\prime} .
$$


Then there is a finite set $Q \subset \mathcal{G}_{\mathbf{h}}$ such that we have the following coset decomposition:

$$
\mathcal{G}_{\mathbb{A}}=\bigsqcup_{q \in Q} \mathcal{G} q W_{\mathfrak{m}} .
$$

The rest of the development is left to the reader; some technically more intricate results in this connection are explained in [D98].

2. Theta correspondence. In this section we generalize the theta lift constructed in [D99] to adelic forms. We first recall the relevant facts from that paper. Let $v \in \delta$ and let $u$ be the extension of $v$ in $\iota$. Then we have

$$
\left(B_{E}\right)_{v}=\left(B_{E}\right)_{u} \times\left(B_{E}\right)_{\tau u}=B_{v} \times B_{v}=\mathrm{M}_{2}(\mathbb{R}) \times \mathrm{M}_{2}(\mathbb{R}) .
$$

Consequently, for $x, y \in B_{v}$ we have $(x, y)^{\tau}=(y, x)$. Thus we further identify

$$
V_{v}=\left\{\left(x,-x^{*}\right) \mid x \in B_{v}\right\}=B_{v} .
$$

When $v \in \delta^{\prime}$, a similar consideration leads to the conclusion $\left(B_{E}\right)_{v}=\mathbb{H} \times \mathbb{H}$.

Denote by $S$ the $F$-valued symmetric form attached to $2 \mathrm{~N}$, i.e.,

$$
S(x, y)=\operatorname{Tr}\left(x y^{*}\right), \quad \forall x, y \in V .
$$

Then we readily see that $S_{v}$ has signature $(2,2)$ if $v \in \delta$, and signature $(4,0)$ if $v \in \delta^{\prime}$. In view of our identifications, we see that for $v \in \delta$ we have

$$
\mathrm{N}(x)=\operatorname{det}(x), \quad S[x]=S(x, x)=2 \operatorname{det}(x), \quad S(x, y)=x y^{*}+y x^{*},
$$

where the symbol * above is now simply the canonical involution for $\mathrm{M}_{2}(\mathbb{C})$.

The theta function is defined by following the standard construction given in, for example, [Sh80]. In our setting its explicit construction is as follows. Let $\xi \in V$ and $w \in \mathbb{C}^{\zeta}$. We define an element $[\xi, w]$ of $\mathbb{C}^{\delta}$ by putting

$$
[\xi, w]_{v}=[\xi, w]_{u}=\left[\xi_{u} ; w_{u}, w_{\tau u}\right]=\left(\begin{array}{ll}
-1 & w_{u}
\end{array}\right) \xi_{u}\left(\begin{array}{c}
w_{\tau u} \\
1
\end{array}\right)
$$

for all $v \in \delta$. We also define, for $\xi, w$ as above and $z \in \mathbb{H}^{\mathbf{a}}$, an element of $\mathbb{C}^{\mathbf{a}}$ in the following manner. At each $v \in \mathbf{a}$, put

$$
\begin{aligned}
& R[\xi, z, w]_{v} \\
& \quad= \begin{cases}\mathrm{N}\left(\xi_{u}\right) \bar{z}_{v} & \text { if }\left.u\right|_{F}=v \in \delta^{\prime}, \\
\mathrm{N}\left(\xi_{u}\right) z_{v}+i \operatorname{Im}\left(z_{v}\right)\left|[\xi, w]_{v}\right|^{2}\left(\operatorname{Im}\left(w_{u}\right) \operatorname{Im}\left(w_{\tau u}\right)\right)^{-1} & \text { if }\left.u\right|_{F}=v \in \delta .\end{cases}
\end{aligned}
$$

Then a positive definite form majorizing $S$ is given by

$$
\begin{aligned}
P_{v}[\xi ; w]=2 \mathrm{~N}(\xi)+\operatorname{Im}\left(w_{1}\right)^{-1} \operatorname{Im}\left(w_{2}\right)^{-1}\left|\left[\xi ; w_{1}, w_{2}\right]\right|^{2}, \\
\forall v \in \delta, \forall \xi \in V_{v}, \forall w=\left(w_{1}, w_{2}\right) \in H \times H .
\end{aligned}
$$

Let $\mathcal{P}_{\delta^{\prime}}=\bigotimes_{v \in \delta^{\prime}} \mathcal{P}_{v}$, where $\mathcal{P}_{v}$ is the space of $S_{v}$-harmonic homogeneous polynomial functions on $V_{v}$ of degree $k_{v}-2$ for every $v \in \delta^{\prime}$. (We also consider $v$ as an element of $\eta^{\prime}$.) Then, viewing $V$ as embedded into $\prod_{v \in \delta^{\prime}} V_{v}$, we can 
define a representation of $\left(B_{E}\right)_{\mathbf{a}}$ on $\mathcal{P}_{\delta^{\prime}}$ which extends the representation $\sigma$ in (1). This representation will again be denoted by $\sigma$. Finally, denote by $\mathcal{L}(V)$ the Schwartz-Bruhat space of $V_{\mathbf{h}}$, the finite part of $V_{\mathbb{A}}$. Alternatively, we may describe $\mathcal{L}(V)$ as the space of locally constant functions on $V$, identifying an element in the Schwartz-Bruhat space with its restriction to $V$. Recall that a locally constant function $C$ on $V$ is characterized by the existence of lattices $L$ and $M$ such that $C$ vanishes off $L$ and that $C(v+m)=C(v)$ for all $m \in M$.

If $C \in \mathcal{L}(V)$ and an element $r \in F$ is chosen such that $r_{v}>0$ for $v \in \delta$ and $r_{v}<0$ for $v \in \delta^{\prime}$, then we define an $\operatorname{End}(\mathcal{X})$-valued function $\theta(z, w ; C, r)$ on $H^{\mathbf{a}} \times H^{\zeta}$ as follows:

$$
\begin{aligned}
\theta(z, w ; C, r)= & \operatorname{Im}(z)^{\delta} \operatorname{Im}(w)^{-k \eta-\tau(k \eta)} \\
& \times \sum_{\xi \in V} C(\xi)[\xi, \bar{w}]^{k \eta} \sigma(\xi) \mathbf{e}_{F}(r R[\xi, z, w]) .
\end{aligned}
$$

Here $\xi, z, w$ are as above, and the notation $\mathbf{e}_{F}(x)$ means $\exp \left(2 \pi i \sum_{v \in \mathbf{a}} x_{v}\right)$.

We shall later need explicit transformation formulas for $\theta(z, w ; C, r)$. The behavior of $\theta(z, w)$ under the action of $\mathcal{G}_{+}^{\prime}$ on the variable $w$ can be determined by relatively straightforward computations. In particular, we have

$$
\theta(z, w ; C, r) \|_{k+\tau k} \alpha=\theta\left(z, w ; C^{\alpha}, \mathrm{N}^{\prime}(\alpha) r\right), \quad \forall \alpha \in \mathcal{G}_{+}^{\prime},
$$

where $C^{\alpha}(\xi)=\mathrm{N}^{\prime}(\alpha)^{k / 2-\eta^{\prime}} C\left(\alpha \xi \alpha^{* \tau}\right)$, and if $\alpha \in \mathcal{G}_{+}^{\prime}$ and $\mathrm{N}(\alpha) \in F^{\times}$, then

$$
\theta(z, w ; C, r) \|_{k+\tau k} \alpha=\theta\left(z, w ; C_{\alpha}, r\right),
$$

where $C_{\alpha}(\xi):=C\left(\alpha \xi \alpha^{-\tau}\right)$. As for the action of $\mathrm{SL}_{2}(F)$ on the variable $z$ of our theta function, we can specialize a theorem of Shimura's in [Sh93] to our setting. We summarize the properties we shall use later as follows. Write $G=\mathrm{SL}_{2}(F)$. Every $\gamma \in G_{\mathbb{A}}$ gives rise to a $\mathbb{C}$-linear automorphism of $\mathcal{L}(V)$, which we denote by $(\gamma, C) \mapsto \gamma C$, such that the following properties hold:

(TFa) $j(\gamma, \bar{z})^{-k} \theta(\gamma z, w ; \gamma C, r)=\theta(z, w ; C, r), \quad \forall \gamma \in G$.

(TFb) $(\gamma \delta) C=\gamma(\delta C), \quad \forall \gamma, \delta \in G_{\mathbb{A}}$.

(TFc) For every $C$, there exists a congruence subgroup $\Gamma$ of $G$, such that

$$
\gamma C=C, \quad \forall \gamma \in \Gamma .
$$

(TFd) If $\beta \in G_{\mathbf{h}}$ and $c_{\beta}=0$, then

$$
(\beta C)(x)=\left|a_{\beta}\right|_{\mathbb{A}}^{2} \omega_{\mathbf{h}}\left(a_{\beta}\right) \mathbf{e}_{\mathbf{h}}\left(r \mathrm{~N}(x) a_{\beta} b_{\beta}\right) C\left(x a_{\beta}\right),
$$

where $\omega$ denotes the Hecke character of $F$ corresponding to $E$, and $\mathbf{e}_{\mathbf{h}}(y):=\mathbf{e}_{\mathbb{A}}\left(y_{\mathbf{h}}\right)$. 
(TFe) For every $p \in F, p \gg 0$,

$$
p^{k / 2} \theta(p z, w ; C, r)=\theta\left(z, w ; p^{\delta+k / 2} C, p r\right) .
$$

Properties (TFa) through (TFd) correspond to items (0), (2), (4), and (5) of Theorem 3.2 in [Sh93], while (TFe) follows from an easy calculation. A more detailed discussion can be found in [D99].

Let $h \in \mathcal{S}_{k+\tau k}\left(B_{E}\right)$. Because of (11), we may consider the inner product of the theta function $\theta(z, w ; C, r)$ with $h$, which we denote as $I(z ; C, r ; h)$ :

$$
I(z ; C, r ; h)=\langle\theta(z, w ; C, r), h(w)\rangle .
$$

Take a congruence subgroup $\Delta \subset \mathcal{G}_{1}^{\prime}$ such that $h \| \gamma=h$ and $C\left(\gamma \xi \gamma^{-\tau}\right)=$ $C(\xi)$ for all $\gamma \in \Delta$, then we have

$$
I(z ; C, r ; h)=\operatorname{vol}(D)^{-1} \int_{D} \operatorname{Tr}\left(\overline{{ }^{t} \theta(z, w ; C, r)} h(w)\right) \operatorname{Im}(w)^{k \eta+\tau(k \eta)} d_{H}^{\zeta} w,
$$

where $D=\Delta \backslash H^{\zeta}$. This integral is convergent. Indeed, in the non-cocompact case $\mathrm{GL}_{2}(E)=\mathcal{G}^{\prime}, \theta(z, w ; C, r)$ is slowly increasing at every cusp. When $C \in \mathcal{L}(V)$ and $r \in F$ are understood, we sometimes write $I(z, h)$ for the sake of notational simplicity. As a function of $z$, this belongs to $\mathcal{S}_{k}\left(\mathrm{SL}_{2}(F)\right)$, as shown in Theorem 2.4 of [D99]. The following formula is easy to verify:

$$
I(z ; C, r ; h) \|_{k} \gamma=I\left(z ; \gamma^{-1} C, r ; h\right), \quad \forall \gamma \in G .
$$

In order to develop the theta correspondence in the adelic setting, we first need some technical preparation. Observe that the properties of $\mu_{v}$ : $\left(B_{E}\right)_{v} \rightarrow \mathrm{M}_{2}\left(E_{v}\right)$ in Section 1 imply that

$$
\mu_{v}\left(V_{v}\right)=\left\{\left(\begin{array}{cc}
a & b \\
c & -a^{\tau}
\end{array}\right) \mid b, c \in F_{v}, a \in E_{v}\right\} \quad \text { if } v \nmid \mathfrak{d}_{B} .
$$

Let us take a Hecke character $\psi_{1}$ of $F$ such that the conductor of $\psi_{1}$ is prime to $\mathfrak{d}_{B}$ and such that

$$
\psi_{1 \mathbf{a}}(x)=\operatorname{sgn}\left(x_{\mathbf{a}}\right)^{k}\left|x_{\mathbf{a}}\right|^{2 i \kappa},
$$

where $\kappa \in \mathbb{R}^{\mathbf{a}}$, and $\|\kappa\|=0$. This choice is natural in view of Section 1 . We have used a lower case letter here since the two fields $E$ and $F$ have to be considered simultaneously. We now consider a special type of $C$.

Proposition 2.1. There exists $C \in \mathcal{L}(V)$ satisfying the following properties:

$$
\begin{gathered}
r \mathrm{~N}(\xi) \in \mathfrak{g} \quad \text { if } C(\xi) \neq 0 . \\
C(s x)=\psi_{1}(s) C(x), \quad \forall s \in \prod_{v \in \mathbf{h}} \mathfrak{g}_{v}^{\times} . \\
C\left(w x w^{* \tau}\right)=\psi_{1 \mathfrak{n}}\left(\mathrm{N}_{E / F}\left(a_{w}\right)\right) C(x), \quad \forall w \in W_{\mathfrak{m}}^{\prime} .
\end{gathered}
$$


Here $\mathfrak{n}:=\mathfrak{m} \cap F$, and $\mathfrak{m}$ is an integral ideal in $E$, such that $\mathfrak{m}$ is divisible by the conductor of $\psi_{1}$, and such that $\mathfrak{m}^{\tau}=\mathfrak{m}$.

Pr o of. We give an example of such a $C$. Our strategy is similar to that of Shimura in Section 6 of [Sh88]. Take an integral ideal $\mathfrak{b}$ in $F$ such that it is prime to $\mathfrak{d}_{B}$ and divisible by the conductor of $\psi_{1}$. For every $v \in \mathbf{h}$, let $\varepsilon_{v} \in B_{v}^{E}$ be an element such that $\varepsilon_{v}=1$ if $v \mid \mathfrak{d}_{B}$ and $\mu_{v}\left(\varepsilon_{v}\right)=\operatorname{diag}\left[1, e_{v}\right]$ if $v \nmid \mathfrak{d}_{B}$. Here $e_{v}$ is an element of $F_{v}$ such that $e_{v} \mathfrak{g}_{v}=\mathfrak{b d}_{v}$. Given $v \in \mathbf{h}$, we define the $v$-component of $C, C_{v}$, as follows:

(a) $C_{v}$ is the characteristic function of $V_{v} \cap \varepsilon_{v} \mathfrak{o}_{v} \varepsilon_{v}^{-1}$ if $v \nmid \mathfrak{b}$.

(b) If $v \mid \mathfrak{b}$, then $C_{v}(\alpha) \neq 0$ only if $\alpha \in \varepsilon_{v} \mathfrak{o}_{v} \varepsilon_{v}^{-1}$ and $b \mathfrak{b} \mathfrak{d}_{v}=\mathfrak{g}_{v}$, in which case $C(\alpha)=\psi_{1 v}\left(b e_{v}\right)$, where $b$ is the $b$-entry of $\mu_{v}(\alpha)$. Define $C$ by the following formula:

$$
C(x)=\prod_{v \in \mathbf{h}} C_{v}\left(x_{v}\right), \quad x \in V_{\mathbf{h}} .
$$

Then the properties (17)-(19) follow from direct, though rather long, computations. We omit the details here to save space. Note that if $r \in \mathfrak{g}$, then $C$ satisfies the properties with any multiple $\mathfrak{m}$ of $\mathfrak{b g}^{E}$ such that $\mathfrak{m}^{\tau}=\mathfrak{m}$.

Following Shimura, we shall call $C$ a standard function of type $\left(\mathfrak{b}, \psi_{1}\right)$.

We insert here some more notation. Define $G=\mathrm{SL}_{2}(F), \widetilde{G}=\mathrm{GL}_{2}(F)$. For two fractional ideals $\mathfrak{x}$ and $\mathfrak{y}$ in $F$ such that $\mathfrak{x} \mathfrak{y} \subset \mathfrak{g}$, write $\mathfrak{o}[\mathfrak{x}, \mathfrak{y}]=\{x \in$ $\left.\mathrm{M}_{2}(F) \mid a_{x} \in \mathfrak{g}, b_{x} \in \mathfrak{x}, c_{x} \in \mathfrak{y}, d_{x} \in \mathfrak{g}\right\}$. We then put, for an integral ideal $\mathfrak{n}$,

$$
\widetilde{D}_{\mathfrak{n}}=\widetilde{D}\left[\mathfrak{d}^{-1}, \mathfrak{n} \mathfrak{d}\right]=\widetilde{G}_{\mathbf{a}+} \prod_{v \in \mathbf{h}} \mathfrak{o}\left[\mathfrak{d}^{-1}, \mathfrak{n} \mathfrak{d}\right]_{v}^{\times}
$$

Further, we put $D_{\mathfrak{n}}=G_{\mathbb{A}} \cap \widetilde{D}_{\mathfrak{n}}, \widetilde{\Gamma}=\widetilde{G} \cap \widetilde{D}_{\mathfrak{n}}, \Gamma=G \cap D_{\mathfrak{n}}$.

Let $C \in \mathcal{L}(V)$ be a standard function of type $\left(\mathfrak{b}, \psi_{1}\right)$, and let $p \in \mathcal{G}_{\mathbf{h}}$. We define another locally constant function $C^{(p)} \in \mathcal{L}(V)$ as follows:

$$
C^{(p)}(x)=C\left(p^{-1} x p^{\tau}\right) .
$$

Proposition 2.2. The locally constant function $C^{(p)}$ satisfies (17), (18), and

$$
C^{(p)}\left(y x y^{* \tau}\right)=\psi_{1 \mathfrak{n}}\left(\mathrm{N}_{E / F}\left(a\left(p^{-1} y p\right)\right)\right) C^{(p)}(x), \quad \forall y \in p W_{\mathfrak{m}}^{\prime} p^{-1} .
$$

Moreover, we have

$$
\gamma C^{(p)}=\omega_{\mathfrak{c}}\left(a_{\gamma}\right) \psi_{1 \mathfrak{f}}\left(a_{\gamma}\right) C^{(p)}, \quad \forall \gamma \in \Gamma .
$$

Here, $\omega$ is the Hecke character of $F$ corresponding to $E, \mathfrak{c}$ is the conductor of $\omega$, and $\mathfrak{f}$ is the conductor of $\psi_{1}$. Finally, $\mathfrak{n}$ is a suitable multiple of $\mathfrak{c} \cap \mathfrak{f}$, which can be defined as $\mathfrak{n}=\mathfrak{f} \cap \mathfrak{c} \cap \mathfrak{t}$, with $\mathfrak{t}$ defined to be $\mathfrak{t}_{v}=$ (4) if $v \mid 2 \mathfrak{g}+\mathfrak{d}_{B}+\mathfrak{c}$, and $\mathfrak{t}_{v}=\left(\mathfrak{d}_{B}\right)_{v}$ otherwise. 
Indeed, all the assertions except for (22) follow from straightforward computations. As for (22), we specialize Proposition 3b.2 of [Sh93] to our setting. Let $S$ in that paper be $2 r \mathrm{~N}$, and $\mathfrak{x}$ there be $2 \mathfrak{g}$. Then one checks easily that the conditions of that proposition are all satisfied, and the assertion there translates into our (22).

For the rest of this section, let $C, C^{(p)}, \mathfrak{m}$, and $\mathfrak{n}$ be fixed as in Propositions 2.1 and 2.2. Set

$$
\psi=\psi_{1} \omega \quad \text { and } \quad \Phi=\psi_{1} \circ \mathrm{N}_{E / F} .
$$

Now an application of (TFa) yields

$$
j(\gamma, \bar{z})^{-k} \theta\left(\gamma z, w ; C^{(p)}, r\right)=\omega_{\mathfrak{c}}\left(a_{\gamma}\right) \psi_{1 \mathfrak{f}}\left(a_{\gamma}\right)^{-1} \theta\left(z, w ; C^{(p)}, r\right), \quad \forall \gamma \in \Gamma .
$$

Take $\gamma=\operatorname{diag}\left[b, b^{-1}\right]$ with any $b \in \mathfrak{g}^{\times}$, we then obtain

$$
\theta\left(b^{2} z, w ; C^{(p)}, r\right)=|b|^{2 i \kappa-k} \theta\left(\gamma z, w ; C^{(p)}, r\right) .
$$

Put

$$
U_{+}=\left\{a \in \mathfrak{g}^{\times} \mid a \gg 0\right\},
$$

and let $U$ be a subgroup of $U_{+}$of finite index contained in $\left\{b^{2} \mid b \in \mathfrak{g}^{\times}\right\}$. We define

$$
\Theta\left(z, w ; C^{(p)}, r\right)=\left[U_{+}: U\right]^{-1} \sum_{a \in U_{+} / U} a^{k / 2-i \kappa} \theta\left(a z, w ; C^{(p)}, r\right) .
$$

This definition is independent of the choice of $U$. It is straightforward to check that

$$
\begin{aligned}
j(\gamma, \bar{z})^{-k} \Theta\left(\gamma z, w ; C^{(p)}, r\right) \\
\quad=\psi_{\mathfrak{n}}\left(a_{\gamma}\right)^{-1} \mathrm{~N}(\gamma)^{-i \kappa} \Theta\left(z, w ; C^{(p)}, r\right), \quad \forall \gamma \in \widetilde{\Gamma} .
\end{aligned}
$$

Given an adelic automorphic form $\mathbf{g} \in \mathcal{S}_{k+\tau k}\left(\mathfrak{m}, \Phi ; B_{E}\right)$, let the index set $Q$ be defined as in (6). Then the theta lift is defined by the following formula:

$$
I(z ; C, r ; \mathbf{g})=\|Q\|^{-1} \sum_{q \in Q}\left\langle\Theta\left(z, w ; C^{(q)}, r\right), g_{q}\right\rangle,
$$

where $g_{q}$ is determined by $\mathbf{g}$ and $q$, as in condition (c) of the definition of adelic forms. This is independent of the choice of representatives $q$ in $Q$.

TheOREm 2.3. As a function of $w, \Theta\left(z, w ; C^{(p)}, r\right)$ belongs to $C\left(\Delta_{p}^{\prime}, \Phi_{\mathfrak{m}}, \kappa\right)$, where $\kappa$ is an element of $\mathbb{R}^{J(E)}$ such that $|x|^{i \kappa}=\left|\mathrm{N}_{E / F}(x)\right|^{i \kappa}$ for $x \in E_{\mathbf{a}}^{\times}$. As a function of $z, I(z ; C, r ; \mathbf{g})$ belongs to $\mathcal{S}_{k}\left(\widetilde{\Gamma}, \psi_{\mathfrak{n}}, \kappa\right)$.

This follows from (23), (10), and (TFe). We leave the details of computation to the reader.

3. Hecke operators and theta correspondence. In this section we consider the behavior of automorphic forms under the theta correspondence 
and the action of Hecke operators. The main theorem gives an explicit "commutativity" property analogous to the ones in Shimura's papers [Sh82], Section 3, and [Sh88], Section 6. Starting from a form $\mathbf{g} \in \mathcal{S}_{k+\tau k}\left(\mathfrak{m}, \Phi, B_{E}\right)$, we need to consider the theta lift of its image under a Hecke operator (with respect to $E$ ), and compare the result with the image under a corresponding Hecke operator (with respect to $F$ ) of the theta lift of $\mathbf{g}$. Since it is our purpose to investigate this situation explicitly, a certain amount of technical calculation and "bookkeeping" is inevitable. To keep clear the outlines of our ideas, we point out here that the following items will be considered in order: certain special Hecke operators on $\mathrm{GL}_{2}(F)$; their action on the theta lift of an automorphic form; Hecke operators on $\mathcal{G}_{\AA}^{\prime}$; the theta lift of the image of a form under such a Hecke operator. The last theorem combines all these considerations to give the main result of this paper.

To consider the Hecke operators on $\mathrm{GL}_{2}(F)$, we specialize the discussion in the first sections of [Sh91] to our situation. Following Shimura's approach there, we formulate everything in terms of $\mathrm{SL}_{2}(F)$ as much as possible. We assume that the reader is familiar with Hilbert modular forms. Thus let $k \in \mathbb{Z}^{\mathbf{a}}$ be an integral weight, $\mathfrak{n}$ be an integral ideal in $F$, and $\Psi$ be a Hecke character such that $\Psi_{\mathbf{a}}(x)=\operatorname{sgn}\left(x_{\mathbf{a}}\right)^{k}\left|x_{\mathbf{a}}\right|^{2 i \lambda}$, where $\lambda \in \mathbb{R}^{\mathbf{a}}$ with $\|\lambda\|=0$. Then we have the space of adelic automorphic forms $\mathcal{S}_{k}(\mathfrak{n}, \Psi)$. For $p \in \widetilde{G}_{\mathbb{A}}$, we define $\widetilde{\Gamma}_{p}=\widetilde{G} \cap p \widetilde{D}_{\mathfrak{n}} p^{-1}$. Let $\phi$ be a character coinciding with $\Psi$ on $(\mathfrak{g} / \mathfrak{n})^{\times}$. There exists a finite set $Q \subset \widetilde{G}_{\mathbf{h}}$ such that $\widetilde{G}_{\mathbb{A}}=\bigsqcup_{q \in Q} \widetilde{G} q \widetilde{D}_{\mathfrak{c}}$. Then there is an embedding

$$
\mathcal{S}_{k}(\mathfrak{n}, \Psi) \hookrightarrow \prod_{q \in Q} \mathcal{S}_{k}\left(\widetilde{\Gamma}_{q}, \phi, \lambda\right),
$$

where for each $q \in Q$ a form $f_{q}$ is defined by the equation

$$
\mathbf{f}(q y)=\Psi(\operatorname{det}(q)) \operatorname{det}(y)^{i \lambda}\left(f_{q} \|_{k} y\right)(\mathbf{i}), \quad \forall y \in \widetilde{G}_{\mathbf{a}+} .
$$

From now on we identify $\mathbf{f}$ and $\left(f_{q}\right)_{q \in Q}$.

Let $Y$ be the subset of $\widetilde{G}_{\mathbb{A}}$ consisting of all elements $y$ such that $y_{v} \in$ $\mathfrak{o}\left[\mathfrak{d}^{-1}, \mathfrak{n} \mathfrak{d}\right]_{v}$ and $a\left(y_{v}\right) \in \mathfrak{g}_{v}^{\times}$for every $v \mid \mathfrak{n}$. Given $y_{0} \in Y$, we have a coset decomposition $\widetilde{D}_{\mathfrak{n}} y_{0} \widetilde{D}_{\mathfrak{n}}=\bigsqcup_{w \in W} \widetilde{D}_{\mathfrak{n}} w$, where $W$ is a finite subset of $\widetilde{G}_{\mathbf{h}}$. More precisely, it is a complete set of representatives of $\left(\widetilde{D}_{\mathfrak{n}} \cap \widetilde{G}_{\mathbf{h}}\right) \backslash\left(\widetilde{D}_{\mathfrak{n}} y_{0} \widetilde{D}_{\mathfrak{n}}\right.$ $\cap \widetilde{G}_{\mathbf{h}}$ ).

Let $\mathbf{f}=\left(f_{q}\right)_{q \in Q}$. We define

$$
\left(\mathbf{f} \mid \widetilde{D}_{\mathfrak{n}} y_{0} \widetilde{D}_{\mathfrak{n}}\right)(x)=\sum_{w \in W} \phi\left(a_{w}^{-1}\right) \mathbf{f}\left(x w^{*}\right), \quad \forall x \in \widetilde{G}_{\mathbb{A}} .
$$

Here $w^{*}$ is the main involution of $w$ as usual. If we write $\mathbf{f} \mid \widetilde{D}_{\mathfrak{n}} y_{0} \widetilde{D}_{\mathfrak{n}}=$ $\left(f_{q}^{\prime}\right)_{q \in Q}$, then the $f_{q}^{\prime}$ are related to the $f_{q}$ as follows. Given $q \in Q$, there 
exists a unique $p \in Q$ and an element $\alpha_{0} \in \widetilde{G}$ such that $q y_{0} \in \alpha_{0} p \widetilde{D}_{\mathfrak{n}}$. Then we may write $\widetilde{\Gamma}_{q} \alpha_{0} \widetilde{\Gamma}_{p}=\bigsqcup_{\alpha \in A} \widetilde{\Gamma}_{q} \alpha$ for some finite set $A$, and

$$
f_{p}^{\prime}=\sum_{\alpha \in A} \phi\left(a\left(q^{-1} \alpha p\right)\right)^{-1} \operatorname{det}(\alpha)^{-i \lambda} f_{q} \|_{k} \alpha .
$$

Given $f \in \mathcal{S}_{k}(\Gamma, \phi, \lambda)$, we can define a function $f_{\mathbb{A}}$ on $G_{\mathbb{A}}$ by

$$
f_{\mathbb{A}}(\alpha w)=\phi\left(a_{w}\right)^{-1}\left(f \|_{k} w\right)(\mathbf{i}), \quad \forall \alpha \in G, \forall w \in D_{\mathfrak{n}} .
$$

The symbols $\Gamma$ and $D_{\mathfrak{n}}$ have been defined in Section 2 . We then have

$$
f_{\mathbb{A}}(\alpha x w)=\phi\left(a_{w}\right)^{-1} j(w, \mathbf{i})^{-k} f_{\mathbb{A}}(x), \quad \forall \alpha \in G, \forall x \in G_{\mathbb{A}}, \forall w \in D_{\mathfrak{n}} .
$$

In fact, the mapping $f \mapsto f_{\mathbb{A}}$ is an injection of $\mathcal{S}_{k}(\Gamma, \phi, \lambda)$ into the space of all functions $g$ on $G_{\mathbb{A}}$ satisfying equation (28) with $f_{\mathbb{A}}$ replaced by $g$. Suppose that $\mathbf{f} \in \mathcal{S}_{k}(\mathfrak{c}, \Psi)$. Consider the form $f_{1} \in \mathcal{S}_{k}(\widetilde{\Gamma}, \phi, \lambda)$ as in $(24)$. Then the restriction of $\mathbf{f}$ to $G_{\mathbb{A}}$ coincides with $\left(f_{1}\right)_{\mathbb{A}}$ as defined by $(27)$ : $\left.\mathbf{f}\right|_{G_{\mathbb{A}}}=\left(f_{1}\right)_{\mathbb{A}}$.

Let $y \in G_{\mathbb{A}} \cap F_{\mathbb{A}}^{\times} Y$. Then we can find a finite subset $W \subset G_{\mathbf{h}}$ such that we simultaneously have $\widetilde{D}_{\mathfrak{n}} y \widetilde{D}_{\mathfrak{n}}=\bigsqcup_{w \in W} \widetilde{D}_{\mathfrak{n}} w$ and $D_{\mathfrak{n}} y D_{\mathfrak{n}}=\bigsqcup_{w \in W} D_{\mathfrak{n}} w$. Furthermore, there exists an element $\alpha_{0}$ and a finite subset $I$ of $G$ such that $D_{\mathfrak{n}} y D_{\mathfrak{n}}=D_{\mathfrak{n}} \alpha_{0} D_{\mathfrak{n}}=D_{\mathfrak{n}} \alpha_{0} \Gamma=\bigsqcup_{\iota \in I} D_{\mathfrak{n}} \iota$ and $\Gamma \alpha_{0} \Gamma=\bigsqcup_{\iota \in I} \Gamma \iota$ hold simultaneously. Let $g$ be a mapping on $G_{\mathbb{A}}$ such that $g$ satisfies (28) with the symbol $f_{\mathbb{A}}$ replaced by $g$. Then, given $y \in G_{\mathbb{A}} \cap F_{\mathbb{A}}^{\times} Y$ and $W$ as above, we define

$$
\left(g \mid D_{\mathfrak{n}} y D_{\mathfrak{n}}\right)(x)=\sum_{w \in W} \phi\left(a_{w}\right)^{-1} g\left(x w^{-1}\right), \quad \forall x \in G_{\mathbb{A}} .
$$

Because of the simultaneous decompositions shown above, if $g$ is the restriction of some $\mathbf{g} \in \mathcal{S}_{k}(\mathfrak{c}, \Psi)$ to $G_{\mathbb{A}}$, then our definition here is consistent with (25).

Now let $f \in \mathcal{S}_{k}(\Gamma, \phi, \lambda)$. Then, with the $\alpha_{0}$ and $I$ given above, we define another element $f \mid \Gamma \alpha_{0} \Gamma$ of $\mathcal{S}_{k}(\Gamma, \phi, \lambda)$ by

$$
f \mid \Gamma \alpha_{0} \Gamma=\sum_{\iota \in I} \phi\left(a_{\iota}\right)^{-1} f \|_{k} \iota .
$$

Then it turns out that

$$
\left(f \mid \Gamma \alpha_{0} \Gamma\right)_{\mathbb{A}}=f_{\mathbb{A}} \mid D_{\mathfrak{n}} y D_{\mathfrak{n}} .
$$

We shall from now on use the same symbol $D_{\mathfrak{n}} y D_{\mathfrak{n}}$ to denote the double coset operation on $f$ as well as on $f_{\mathbb{A}}$. That is, we shall write $f \mid D_{\mathfrak{n}} y D_{\mathfrak{n}}$ for $f \mid \Gamma \alpha_{0} \Gamma$.

Let us now apply the Hecke operators to $I(z ; C, r ; h)$. To simplify notation let us write $D_{\mathfrak{n}}$ as $D$. Note that if $C \in \mathcal{L}(V)$ satisfies

$$
\gamma C=\phi\left(a_{\gamma}\right) C
$$

for all $\gamma \in \Gamma$, then $I(z ; C, r ; h) \in \mathcal{S}_{k}(\Gamma, \phi)$. 
Proposition 3.1. Let $y_{0} \in G_{\mathbf{h}} \cap Y$ and let $f(z)=\langle\theta(z, w ; C, r), h(w)\rangle$ with $C$ satisfying (31). Let $D y_{0} D=\bigsqcup_{u} D u$, where $u$ runs through a finite subset of $G_{\mathbf{h}}$ as usual. Then

$$
f_{\mathbb{A}} \mid D y_{0} D=\left\langle\theta\left(z, w ; C^{\prime}, r\right), h(w)\right\rangle_{\mathbb{A}},
$$

where

$$
C^{\prime}=\sum_{u} \phi\left(a\left(u_{\mathfrak{n}}\right)\right) u^{-1} C .
$$

Moreover, the assertion remains valid if we substitute $\theta$ there by $\Theta$.

Pr o of. To prove the first assertion, we specialize a theorem of Shimura to our situation. Let us define a function $\theta_{\mathbb{A}}(x, C)$ on $G_{\mathbb{A}}$ by

$$
\theta_{\mathbb{A}}(\alpha y, C)=\overline{j(y, \mathbf{i})}^{-k} \theta(y(\mathbf{i}), w ; C, r),
$$

for $\alpha \in G, y \in G_{\mathbb{A}}$, and $y C=C$. Then we have $f_{\mathbb{A}}(x)=\left\langle\theta_{\mathbb{A}}(x, C), h\right\rangle$. Proposition 3.6 of [Sh93] now gives the desired result. The second assertion follows directly from the definition of $\Theta$.

Proposition 3.2. Let $y_{0}=\operatorname{diag}\left[\pi^{-1}, \pi\right]$ and $\mathfrak{p}=\pi \mathfrak{g}$ with a prime element $\pi$ of $F_{v}$, where $v \in \mathbf{h}$ and $v \nmid \mathfrak{n}$. Let $C^{\prime}$ be as in Proposition 3.1. Then, for $\xi \in V$ such that $r \mathrm{~N}(\xi) \in \mathfrak{g}$, we have

$$
\begin{aligned}
C^{\prime}(\xi)= & \omega_{v}(\pi) C(\pi \xi)+\omega_{v}(\pi)^{-1} \mathrm{~N}(\mathfrak{p})^{2} C\left(\pi^{-1} \xi\right) \\
& + \begin{cases}{[\mathrm{N}(\mathfrak{p})-1] C(\xi)} & \text { if } r \mathrm{~N}(\xi) \in \mathfrak{p}, \\
-C(\xi) & \text { if } r \mathrm{~N}(\xi) \notin \mathfrak{p} .\end{cases}
\end{aligned}
$$

Moreover, suppose that $C(\xi)=0$ if $r \mathrm{~N}(\xi) \notin \mathfrak{g}$. Then $C^{\prime}$ has the same property.

Proof. We begin with a remark on notation: here we have $\omega(\pi)=$ $\omega_{v}(\pi)=\omega_{\mathbf{h}}(\pi)$. For the sake of notational consistency we will always write $\omega_{v}(\pi)$. For $v \nmid \mathfrak{n}$, the following $\mathrm{N}(\mathfrak{p})^{2}+\mathrm{N}(\mathfrak{p})$ elements form a complete set of representatives for $D \backslash D y_{0} D$ :

$$
\begin{aligned}
\alpha_{b} & =\left(\begin{array}{cc}
1 / \pi & b /(\pi \delta) \\
0 & \pi
\end{array}\right), \quad \forall b \in \mathfrak{g}_{v} / \mathfrak{p}_{v}^{2} \\
\beta_{h} & =\left(\begin{array}{cc}
1 & h /(\pi \delta) \\
0 & 1
\end{array}\right), \quad \forall h \in\left(\mathfrak{g}_{v} / \mathfrak{p}_{v}\right)^{\times}, \\
\gamma & =\left(\begin{array}{cc}
\pi & 0 \\
0 & 1 / \pi
\end{array}\right) .
\end{aligned}
$$

An explanation of this fact can be found, for example, in [Sh90]. Here $\delta$ is an element of $F_{v}$ such that $\delta \mathfrak{g}_{v}=\mathfrak{d}_{v}$. We now calculate $C^{\prime}(\xi)$ by taking these elements as the $u$ in the formula in Proposition 3.1. Thus

$$
C^{\prime}(\xi)=\sum_{b} \alpha_{b}^{-1} C+\sum_{h} \beta_{h}^{-1} C+\gamma^{-1} C .
$$


We can now compute the three summands separately by applying (TFd). We record the final results here:

$$
\begin{aligned}
\sum_{b} \alpha_{b}^{-1} C & =\sum_{b} \mathrm{~N}(\mathfrak{p})^{-2} \omega_{v}(\pi) \mathbf{e}_{\mathbf{h}}\left(r \mathrm{~N}(\xi) \cdot \frac{-b}{\delta}\right) \cdot C(\pi x) \\
= & \begin{cases}\omega_{v}(\pi) C(\pi x) & \text { if } r \mathrm{~N}(\xi) \in \mathfrak{g}_{v} \\
0 & \text { if } r \mathrm{~N}(\xi) \notin \mathfrak{g}_{v}\end{cases} \\
\sum_{h} \beta_{h}^{-1} C & =C(x) \sum_{h} \mathbf{e}_{\mathbf{h}}\left(r \mathrm{~N}(\xi) \cdot \frac{-h}{\pi \delta}\right) \\
& = \begin{cases}C(x)[\mathrm{N}(\mathfrak{p})-1] & \text { if } r \mathrm{~N}(\xi) \in \mathfrak{p}_{v}, \\
-C(x) & \text { if } r \mathrm{~N}(\xi) \notin \mathfrak{p}_{v}\end{cases}
\end{aligned}
$$

and

$$
\gamma^{-1} C=\mathrm{N}(\mathfrak{p})^{2} \omega_{v}(\pi)^{-1} C\left(\pi^{-1} x\right)
$$

Collecting these facts we obtain (32).

Notice that condition (31) implies that our result is independent of the choice of $\pi$.

Suppose $\psi_{1}$ is a Hecke character of $F$ such that $\psi_{1}$ satisfies the condition (16), and such that $\psi_{1}=\phi$ on $\prod_{v \mid \mathfrak{n}} \mathfrak{g}_{v}^{\times}$. Take an element $s$ of $F_{\mathbf{h}}^{\times}$such that $s \mathfrak{g} \subset \mathfrak{g}$. Then we have the following natural consequence of Proposition 3.2:

Proposition 3.3. If $v \mid \mathfrak{n}$ and $r \mathrm{~N}(\xi) \in \mathfrak{g}$, then

$$
C^{\prime}(\xi)=\left(\psi_{1} \omega\right)_{v}(\pi) C(\pi \xi) .
$$

The last assertion of Proposition 3.2 is also valid here.

We now turn our attention to Hecke operators on the adelic automorphic forms. The development essentially parallels what we have just seen. We define a subset $\mathcal{Y}$ of $\mathcal{G}_{\mathbb{A}+}^{\prime}$ as follows:

$$
\begin{aligned}
\mathcal{Y}=\left\{y \in \mathcal{G}_{\mathbb{A}+}^{\prime} \mid y_{v} \in \mathfrak{o}_{1 v}(\forall v \in \mathbf{h}), y_{u} \in \mathfrak{o}_{1 u}^{\times}\left(\text {if } u \mid \mathfrak{d}_{B}^{E}+\mathfrak{m}\right),\right. \\
\left.\mu_{v}\left(y_{v}\right)=\left(\begin{array}{cc}
a & * \\
* & *
\end{array}\right), \text { where } a \in\left(\mathfrak{g}_{u}^{E}\right)^{\times}\left(\text {if } u \mid \mathfrak{m}, u \nmid \mathfrak{d}_{B}^{E}\right)\right\} .
\end{aligned}
$$

For $y_{0} \in \mathcal{Y}$ and $\mathbf{g} \in \mathcal{S}_{k+\tau k}\left(\mathfrak{m}, \Phi, B_{E}\right)$, we have a coset decomposition

$$
W^{\prime} y_{0} W^{\prime}=\bigsqcup_{y \in J} W^{\prime} y
$$

for some finite set $J$. More precisely, $J$ is a complete set of representatives for the quotient space $\left(W^{\prime} \cap \mathcal{G}_{\mathbf{h}}^{\prime}\right) \backslash\left(W^{\prime} y_{0} W^{\prime} \cap \mathcal{G}_{\mathbf{h}}^{\prime}\right)$. We define $\mathbf{g} \mid W^{\prime} y_{0} W^{\prime}$ by

$$
\left(\mathbf{g} \mid W^{\prime} y_{0} W^{\prime}\right)(x)=\sum_{y \in J} \Phi_{\mathfrak{m}}\left(a_{y}\right)^{-1} \mathbf{g}\left(x y^{*}\right), \quad \forall x \in \mathcal{G}_{\mathbb{A}}^{\prime} .
$$


If $y_{0} \in \mathcal{Y}$ and $q \in Q^{\prime}$ are given, there exist an element $\alpha_{0} \in \mathcal{G}^{\prime}$ and a unique $p \in Q^{\prime}$, such that $q y_{0} \in \alpha_{0} p W^{\prime}$. Then there is a coset decomposition

$$
\Delta_{q}^{\prime} \alpha_{0} \Delta_{p}^{\prime}=\bigsqcup_{\alpha \in A} \Delta_{q}^{\prime} \alpha
$$

for some finite set $A$.

If $\mathbf{g}=\left(g_{q}\right)_{q \in Q^{\prime}} \in \mathcal{S}_{k+\tau k}\left(\mathfrak{m}, \Phi, B_{E}\right)$ and $\mathbf{g} \mid W^{\prime} y_{0} W^{\prime}=\left(\widetilde{g}_{q}\right)_{q \in Q^{\prime}}$, then it is straightforward to check that

$$
\widetilde{g}_{p}=\sum_{\alpha \in A} \Phi_{\mathfrak{m}}\left(a\left(q^{-1} y p\right)\right)^{-1} \mathrm{~N}(\alpha)^{-i \kappa} g_{q} \| \alpha .
$$

Because of this fact, given $\left(f_{q}\right)_{q \in Q^{\prime}} \in \prod_{q \in Q^{\prime}} C\left(\Delta_{q}^{\prime}, \Phi_{\mathfrak{m}}, \kappa\right)$, we may define

$$
\left(f_{q}\right) \mid W^{\prime} y_{0} W^{\prime}=\left(\tilde{f}_{q}\right)_{q \in Q^{\prime}},
$$

where the $\widetilde{f}_{q}$ are determined by (35), with $g$ replaced by $f$.

Regarding automorphic forms defined with respect to $\mathcal{G}$, we define operators $W y W$ in an analogous manner. The details will mostly be left to the reader, but we point out here the connection between the operators $W^{\prime} y W^{\prime}$ and $W y W$ for $y \in G_{\mathbb{A}}$. Suppose, therefore, that we are given $y \in G_{\mathbb{A}}$. Consider a coset decomposition

$$
W y W=\bigsqcup_{r} W r
$$

where the $r$ run through a finite subset of $\mathcal{G}_{\mathbf{h}}$. Also, given $q \in Q$, there is (as usual) a unique $p \in Q$ and some $\alpha_{0} \in \mathcal{G}$ such that $q y \in \alpha_{0} p W$. Letting $\Delta_{q}=\Delta_{q}^{\prime} \cap \mathcal{G}$, we can take a coset decomposition

$$
\Delta_{q} \alpha_{0} \Delta_{p}=\bigsqcup_{\alpha \in A} \Delta_{q} \alpha
$$

We then have

$$
W^{\prime} y W^{\prime}=W^{\prime} y W
$$

and more generally,

$$
\Delta_{q}^{\prime} \alpha_{0} \Delta_{p}^{\prime}=\Delta_{q}^{\prime} \alpha_{0} \Delta_{p}=\bigsqcup_{\alpha \in A} \Delta_{q}^{\prime} \alpha .
$$

The interested reader can find a proof in [D98]. In view of this fact, we have

$$
\left(\mathbf{g} \mid W^{\prime} y_{0} W^{\prime}\right)(x)=\sum_{r} \Phi_{\mathfrak{m}}\left(a_{r}\right)^{-1} \mathbf{g}\left(x r^{*}\right)
$$

with the same $r$ as those in the formula (36). Moreover, we see that (35) is valid in this setting - that is, with respect to the $A$ in (37) — as well. 
From now on, we shall again adopt the notation and conventions made in the last part of Section 2. Recall that by definition

$$
I(z ; C, r ; \mathbf{g})=\|Q\|^{-1} \sum_{q \in Q}\left\langle\Theta\left(z, w ; C^{(q)}, r\right), g_{q}\right\rangle .
$$

Also, for each $q \in Q$, we can find an element $\alpha_{0} \in \mathcal{G}$ and a unique $p \in Q$ such that $q y_{0} \in \alpha_{0} p W$. Let $\Delta_{q} \alpha_{0} \Delta_{p}=\bigsqcup_{\alpha \in A} \Delta_{q} \alpha$. Write $\Theta_{q}(w)=\Theta\left(z, w ; C^{(q)}, r\right)$ and $T_{v}=W_{\mathfrak{m}}^{\prime} y_{0} W_{\mathfrak{m}}^{\prime}$. We recall the following fact from [D98]. Let $\left(f_{q}\right)_{q \in Q} \in$ $\prod_{q \in Q} C\left(\Delta_{q}, \Phi_{\mathfrak{m}}, \kappa\right)$, and define $\left(\widetilde{f}_{q}\right)_{q \in Q}$ by (35) with $g$ there replaced by $f$. Let $\mathbf{g}=\left(g_{q}\right)_{q \in Q} \in \mathcal{S}_{k+\tau k}\left(\mathfrak{m}, \Phi, B_{E}\right)$, and write $\mathbf{g} \mid W y_{0} W=\left(\widetilde{g}_{q}\right)_{q \in Q}$. Suppose $y_{0} \in \mathcal{Y}$ and that $\mathrm{N}\left(y_{0}\right) \mathfrak{g}^{E}$ is prime to $\mathfrak{m}$. Then

$$
\Phi^{*}\left(\mathrm{~N}\left(y_{0}\right) \mathfrak{g}^{E}\right)\left\langle\widetilde{f}_{p}, g_{p}\right\rangle=\left\langle f_{q}, \widetilde{g}_{q}\right\rangle,
$$

so long as the inner products are convergent. Applying this fact to our $\Theta$ and $\mathbf{g}$, we derive

$$
I\left(z ; C, r ; \mathbf{g} \mid T_{v}\right)=\psi(\pi)^{2}\|Q\|^{-1} \sum_{p \in Q}\left\langle\widetilde{\Theta}_{p}, g_{p}\right\rangle,
$$

where $\widetilde{\Theta}_{p}=\sum_{\alpha \in A} \Phi_{\mathfrak{m}}\left(a\left(q^{-1} \alpha p\right)\right)^{-1} \mathrm{~N}(\alpha)^{-i \kappa} \Theta_{q} \| \alpha$. Define

$$
\widetilde{C}(x)=\sum_{y \in W \backslash W y_{0} W} \Phi_{\mathfrak{m}}\left(a_{y}\right)^{-1} \psi_{1}(\mathrm{~N}(y)) C\left(y x y^{-\tau}\right) .
$$

We now use $\left\{\left(q^{-1} \alpha p\right)_{\mathbf{h}} \mid \alpha \in A\right\}$ as the $y$ in $\widetilde{C}$. Then we easily obtain

Proposition 3.4. With the $\widetilde{C}$ in (38) we have

$$
I\left(z ; C, r ; \mathbf{g} \mid T_{v}\right)=\psi\left(\pi_{v}\right)^{2} I(z ; \widetilde{C}, r ; \mathbf{g}) .
$$

We are now ready to present the main theorem of this paper.

Theorem 3.5. Let us write $f(z)=I(z ; C, r ; \mathbf{g})$. Let $Z_{v}=D_{\mathfrak{n}} z_{0} D_{\mathfrak{n}}$, with $z_{0}:=\operatorname{diag}\left[\pi_{v}^{-1}, \pi_{v}\right]$, where $\pi_{v}$ is a prime element of $F_{v}$. Then for almost all primes $v$, we have

$$
\psi\left(\pi_{v}\right)^{-1} I\left(z ; C, r ; \mathbf{g} \mid T_{v}\right)=f \mid Z_{v}+f+\omega\left(\pi_{v}\right) \mathrm{N}_{v} f,
$$

where $\mathrm{N}_{v}=\mathrm{N}\left(\pi_{v} \mathfrak{g}\right)$.

P r o o f. We begin by observing that we have $\left(C^{\prime}\right)^{(q)}=\left(C^{(q)}\right)^{\prime}$. Therefore, by Propositions 3.1 and 3.2, we have

$$
f \mid Z_{v}=I\left(z ; C^{\prime}, r ; \mathbf{g}\right)
$$

with the $C^{\prime}$ there for $v \nmid \mathfrak{n}$. Combining this with Proposition 3.4, we see that (40) is reduced to the verification of the equation

$$
\psi\left(\pi_{v}\right)^{-1} \widetilde{C}=C^{\prime}+C+\omega\left(\pi_{v}\right) \mathrm{N}_{v} C
$$

for almost all $v$. Notice that $I(z ; C, r ; \mathbf{g})$ is anti-C-linear in $C$. 
We can find a finite subset $\mathbf{b}$ of $\mathbf{h}$ such that

$$
C(x)=C_{1}\left(x_{\mathbf{b}}\right) \prod_{v \notin \mathbf{b}} C_{v}\left(x_{v}\right),
$$

where $C_{v}$ is the characteristic function of $V_{v} \cap \varepsilon_{v} \mathfrak{o}_{v} \varepsilon_{v}^{-1}$ with the $\varepsilon_{v}$ as in part (a) of the definition of the standard function, and where $C_{1}$ is a function on $\prod_{v \in \mathbf{b}} V_{v}$. Then, for each $v \notin \mathbf{b}$, we have

$$
C(x)=C_{v}\left(x_{v}\right) C_{v}^{*}\left(x_{v}^{\prime}\right)
$$

with a function $C_{v}^{*}$ on $\prod_{w \neq v} V_{w}$, where we have written the projection of $x$ to that product by $x^{\prime}$.

The $y$ in (38) can be chosen from $\mathcal{G}_{v}$. With such $y$, let us denote by $\widetilde{C}_{v}$ the function on $V_{v}$ given by the right-hand side of (38), but with $C$ there replaced by $C_{v}$. Similarly, we let $C_{v}^{\prime}$ be the function on $V_{v}$ defined by the formula (32) for $C^{\prime}$ in Proposition 3.2, but with $C$ replaced by $C_{v}$.

With these notations in place, we find that the equation (41) is now equivalent to

$$
\psi\left(\pi_{v}\right)^{-1} \widetilde{C}_{v}=C_{v}^{\prime}+C_{v}+\omega\left(\pi_{v}\right) \mathrm{N}_{v} C_{v}, \quad \forall v \notin \mathbf{b} .
$$

Finally, we observe that to prove (42), we may assume that $v \nmid \mathfrak{d}_{B} \mathfrak{m n}$ and that $r$ is a $v$-unit. Also, it is sufficient to consider the set $\left\{x \in V_{v} \mid \mathrm{N}(x) \in\right.$ $\left.\mathfrak{g}_{v}\right\}$.

The rest is computation. In the main we can follow the framework of Shimura's proof of his Theorem 6.7 in [Sh88]. In order not to obscure ideas, we shall outline the computations only in the following case:

We assume that $\pi x \in \mathfrak{o}_{v}, x \notin \mathfrak{o}_{v}^{\prime}$, and $v$ splits in $E$.

For notational simplicity, we shall write $\pi_{v}$ simply as $\pi$ from now on. Take an element $\delta$ of $\mathfrak{g}_{v}$ so that $\delta \mathfrak{g}_{v}=\mathfrak{d}_{v}$. By Proposition 3.2, we have $C_{v}^{\prime}(x)=\omega_{v}(\pi)$. To compute $\widetilde{C}_{v}$, we shall identify $\left(B_{E}\right)_{v}$ with $\mathrm{M}_{2}\left(F_{v} \times F_{v}\right)$ and identify $V_{v}$ with the set $\left\{\left(\begin{array}{cc}a & b \\ c & -a^{\tau}\end{array}\right) \mid b, c \in F_{v}, a \in E_{v}\right\}$ via (15). We define the following symbols:

$$
\mathfrak{p}=\pi \mathfrak{g}_{v}, \quad z=\left(\begin{array}{cc}
\pi & 0 \\
0 & 1
\end{array}\right)
$$

and

$$
w_{i}=\left(\begin{array}{cc}
1 & i / \pi \\
0 & \pi
\end{array}\right), \quad i \in R,
$$

where $R$ is a fixed set of representatives for $\mathfrak{g}_{v} / \mathfrak{p}$. Then there are $[\mathrm{N}(v)+1]^{2}$ elements of the form $\left(w_{i}, w_{j}\right),\left(z, w_{j}\right),\left(w_{i}, z\right)$, and $(z, z)$. We can take these elements as the $y$ in $(38)$.

Note that an element $x$ of $V_{v}$ can be written as $x=\left(u,-u^{*}\right)$, where $u=\left(\begin{array}{cc}a & b / \delta \\ c \delta & d\end{array}\right)$ with $a, b, c$, and $d$ in $F_{v}$. 
We now put

$$
W_{i j}=w_{i} u w_{j}^{-1}, \quad X_{i}=w_{i} u z^{-1}, \quad Y_{i}=z u w_{i}^{-1}, \quad Z=z u z^{-1} .
$$

Then we see that the $[\mathrm{N}(v)+1]^{2}$ elements $y x y^{-\tau}$ are $\left(U, U^{*}\right)$ with $U$ in the set of elements of (44). Finally we make two simple computational observations. First of all, we have

$$
(a+c i)(d-c j)-(a d-b c)=c(b-a j+d i-c i j) ;
$$

and secondly, the $b$-entry of $W_{i j}$ is $(b-a j+d i-c i j) /(\pi \delta)$.

Let us now consider the various cases. If $c \notin \mathfrak{g}_{v}$, then $\pi c$ is a $v$-unit. Under the condition (43), we see that there is a unique $(i, j)$ such that $a+c i \in \mathfrak{g}_{v}$ and $d-c j \in \mathfrak{g}_{v}$. By (45), then, we find that the $W_{i j}$ for this $(i, j)$ is the only element among those in (44) which is contained in $\mathfrak{o}_{v}^{\prime}$. Therefore, by (38), we conclude that $\widetilde{C}(x)=\psi_{1 v}(\pi)$.

Next we consider the case $c \in \mathfrak{g}_{v}$, but $a \notin \mathfrak{g}_{v}$. This time the only possible elements among those in (44) that are contained in $\mathfrak{o}_{v}^{\prime}$ are the $Y_{j}$. Now $b-a j \in \mathfrak{g}_{v}$ for a unique $j$ and we have

$$
d-c j \equiv d-\frac{c b}{a} \equiv \frac{a d-c b}{a} \equiv 0(\bmod \mathfrak{p}) .
$$

Therefore, we see that in fact only the $Y_{j}$ for this particular $j$ belongs to $\mathfrak{o}_{v}^{\prime}$. Therefore, again we have $\widetilde{C}(x)=\psi_{1 v}(\pi)$.

The case $c \in \mathfrak{g}_{v}$ and $d \notin \mathfrak{g}_{v}$ is similar to the case above. The conclusion is that there exists a unique $i$ such that $X_{i} \in \mathfrak{o}_{v}^{\prime}$.

Finally we consider the case where we have $c \in \mathfrak{g}_{v}, a \in \mathfrak{g}_{v}$, and $d \in \mathfrak{g}_{v}$, but $b \notin \mathfrak{g}_{v}$. In this case, $Z$ is the only element in (44) that belongs to $\mathfrak{o}_{v}^{\prime}$. The details can be omitted.

This then verifies (42) in the case specified by (43). Therefore the theorem is proved in that case. For the other cases, the computations have the same flavor; a detailed explanation can therefore be spared.

\section{References}

[D94] Z. L. Dou, On the fundamental periods of Hilbert modular forms, Trans. Amer. Math. Soc. 346 (1994), 147-158.

[D98] —, On adelic automorphic forms with respect to a quadratic extension, Bull. Austral. Math. Soc., to appear.

[D99] -, On a theta correspondence with respect to a quadratic extension, J. Number Theory 79 (1999), 1-18.

[Sh80] G. Shimura, The arithmetic of certain zeta functions and automorphic forms on orthogonal groups, Ann. of Math. 111 (1980), 313-375.

[Sh81I] -, On certain zeta functions attached to two Hilbert modular forms, I, ibid. 114 (1981), 127-164. 
[Sh81II] G. Shimura, On certain zeta functions attached to two Hilbert modular forms, $I I$, ibid. 114 (1981), 569-607.

[Sh82] - The periods of certain automorphic forms of arithmetic type, J. Fac. Sci. Univ. Tokyo Sect. IA 28 (1982), 605-632.

[Sh88] - On the critical values of certain Dirichlet series and the periods of automorphic forms, Invent. Math. 94 (1988), 245-305.

[Sh90] - On the fundamental periods of automorphic forms of arithmetic type, ibid. 102 (1990), 399-428.

[Sh91] —, The critical values of certain Dirichlet series attached to Hilbert modular forms, Duke Math. J. 63 (1991), 557-613.

[Sh93] - On the transformation formulas of theta series, Amer. J. Math. 115 (1993), 1011-1052.

[Sh] —, Two correspondences to Ze-Li Dou, unpublished.

[Y] H. Yoshida, On a conjecture of Shimura concerning periods of Hilbert modular forms, Amer. J. Math. 117 (1995), 1019-1038.

Mathematics Department

P.O. Box 298900

Texas Christian University

Fort Worth, TX 76129, U.S.A.

E-mail: z.dou@tcu.edu 